\title{
Erratum to: Long non-coding RNAs in cancer: implications for personalized therapy
}

\author{
Marianna Vitiello • Andrea Tuccoli • Laura Poliseno
}

Published online: 7 October 2014

(C) International Society for Cellular Oncology 2014

\section{Erratum to: Cell Oncol.}

\section{DOI 10.1007/s13402-014-0180-x}

The following references are missing from the reference list of above mentioned article:

173. D. Mei, H. Song, K. Wang, Y. Lou, W. Sun, Z. Liu, X. Ding, J. Guo, Med. Oncol. 30 (4), 709 (2013). Doi 10.1007/ s12032-013-0709-2

Cross referenced in paragraph 3.2: However, given the number of pseudogenes for which a link to cancer has been proven and given the cancer-specific expression of some of them, this class of ncRNAs is surely about to make its appearance as a useful diagnostic and prognostic tool $[116,121,173]$.

33. E. Pasmant, I. Laurendeau, D. Héron, M. Vidaud, D. Vidaud, I. Bièche, Cancer Res. 67 (8), 3963-3969 (2007).

Cross referenced in paragraph 2.1 : The INK4b-ARFINK4a gene locus is transcribed both in sense and in antisense orientation: in the first case, it encodes for three tumor suppressor genes (INK4b-ARF-INK4a), while in the second case it transcribes the lncRNA ANRIL (antisense non-coding RNA in the INK4 locus) [33].

The online version of the original article can be found at htt://dx.doi.org/ 10.1007/s13402-014-0180-x.

M. Vitiello · A. Tuccoli $\cdot$ L. Poliseno $(\bowtie)$

Oncogenomics Unit, Core Research Laboratory, Istituto Toscano,

Tumori c/o IFC-CNR, via Moruzzi 1, 56124 Pisa, Italy

e-mail: laura.poliseno@gmail.com

\section{Poliseno}

Institute of Clinical Physiology, National Research Council,

Pisa, Italy 